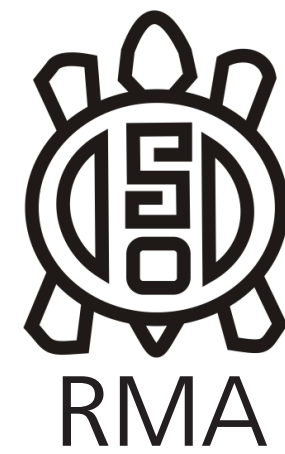

Dossier

\section{Disponibilidad y selección de xilópalos en la costa norte y sur de la Provincia de Chubut, Patagonia Argentina}

\author{
Availability and selection of fossilized wood on the north and south \\ coast of the Province of Chubut, Patagonia Argentina \\ Anahi Banegas* y Julieta Gómez Otero** \\ *Instituto de Diversidad y Evolución Austral (IDEAus-CONICET). Bvard. Brown 2915, \\ Puerto Madryn. E-mail: banegas.anahi@yahoo.com.ar \\ ** Instituto de Diversidad y Evolución Austral (IDEAus-CONICET), Bvard. Brown 2915, \\ Puerto Madryn. E-mail: julietagomezotero@yahoo.com.ar
}

\begin{abstract}
Resumen
La presencia de xilópalos ha sido registrada en distintos contextos arqueológicos de la costa de la provincia de Chubut, aunque su frecuencia es variable y exhiben distintas calidades para la talla. Con el objetivo de explorar la relación entre la frecuencia de artefactos de xilópalo, y la distribución y calidad de las fuentes reconocidas localmente, en este trabajo se analizan xilópalos recuperados en sitios de la costa norte (entre Arroyo Verde y Bahía Cracker, incluida Península Valdés) y la costa sur (entre bahía Camarones y bahía Solano). Los resultados indican un aprovechamiento muy escaso en el sector norte y alto en el sur, lo que sugiere una vinculación estrecha entre la intensidad de uso de los xilópalos y la oferta local: a menor distancia respecto de los afloramientos primarios de excelente calidad se observa un mayor aprovechamiento, y viceversa. Por lo tanto, para la costa sur se infiere obtención directa y para la costa norte adquisición mediante mecanismos indirectos (intercambio) y también aprovechamiento de hallazgos fortuitos de fragmentos o tabletas en los afloramientos locales del Terciario.
\end{abstract}

Palabras clave: Xilópalos; Tecnología lítica; Fuentes; Costa; Patagonia.

\begin{abstract}
The presence of fossilized wood has been recorded in different archaeological contexts of the coast of the province of Chubut, although their frequency is variable and they show different qualities for flaking. In order to explore the relationship between the frequency of fossilized wood artifacts, and the distribution and quality of locally recognized sources, this paper analyzes fossilized wood artifacts recovered from sites on two coastal sectors of Chubut province: the north coast (between Arroyo Verde and Bahía Cracker, including Península Valdés) and the south coast (between Camarones and Bahía Solano). The results indicate a very limited use in the northern sector and high in the south, which suggests a close link between the intensity of use of the fossilized wood and the local supply: the lower distance from the primary outcrops of excellent quality, the more intensive exploitation; and vice versa. Therefore, for the south coast, direct acquisition is hypothesized, while for the north coast is inferred indirect obtaining (by trade) and also exploitation of fragments and small nodules occasionally found in local Tertiary sedimentary outcrops.
\end{abstract}

Keywords: Fossilized Wood; Lithic technology; Sources; Coast; Patagonia.

\section{Introducción}

Para la costa de Patagonia son escasos los estudios sobre el uso de los xilópalos y sus potenciales fuentes de aprovisionamiento (excepto trabajos de Arrigoni y Paleo 1991, Mansur 2008, Ambrústolo 2010, Banegas et al. 2016, entre otros). Esto podría deberse a su baja disponibilidad en los ambientes costeros o a dificultades en su identificación litológica dada la alta variabilidad de soportes, colores, texturas y calidad para la talla que se presentan, en general, en los ambientes litorales.

Estudios previos de caracterización de la base regional de recursos líticos en la costa norte y valle inferior del río Chubut, determinaron una muy escasa representación de los xilópalos en los muestreos $(<1,5 \%)$; la mayoría de los cuales proviene de fuentes secundarias, en especial con morfología de rodado (Gómez Otero et al. 1999; Banegas y Goye 2014; Banegas et al. 2016). Sin embargo, es relativamente superior la presencia 
de los xilópalos en los conjuntos arqueológicos (entre $5 \%$ y $6 \%$ ), destacándose variabilidad en cuanto a la procedencia de las piezas - fuentes primarias y secundarias (guijas, guijarros, tabletas)- y los tamaños, colores y calidad para la talla (Banegas et al. 2016). Para el resto del litoral chubutense no se ha generado información cuantitativa y cualitativa equivalente, excepto para una porción de la costa sur, donde en 2017 se han iniciado investigaciones sistemáticas que incluyen estos estudios, entre otros. Sobre la base de los datos obtenidos hasta el presente, en este trabajo se explora la relación entre la frecuencia de artefactos de xilópalo y la distribución y calidad de las fuentes reconocidas localmente. Se analizan comparativamente dos sectores: la costa norte (entre Arroyo Verde y Bahía Cracker, incluida Península Valdés) y la costa sur entre bahía Camarones y bahía Bustamante (Figura 1).

\section{Oferta de materias primas líticas en las costas norte y sur de la provincia de Chubut}

Se trata de dos tipos de costas con características ambientales y geológicas diferentes. En la costa norte (en adelante $\mathrm{CN}$ ), los únicos afloramientos primarios se encuentran en la costa oeste del golfo San Matías, y corresponden a la Fm. Marifil (Malvicini y Llambías 1974) que ofrece ignimbritas, riolitas y calcedonias (Haller y Massaferro 2001). El resto del sector se caracteriza por poseer rocas sedimentarias de edad Terciaria (Fm. Gaiman y Fm. Puerto Madryn) y abundantes fuentes secundarias, como rodados marinos y Rodados Tehuelches (Haller 1981; Haller 2017). Entre las litologías sobresalen basaltos, riolitas y cuarcitas; en baja proporción, sílices y calcedonias (Banegas et al. 2015). Con relación a los xilópalos, se realizaron 15 muestreos sistemáticos en fuentes primarias y secundarias (Banegas et al. 2016) y también recolecciones asistemáticas en las mesetas inmediatamente adyacentes, a $15 \mathrm{~km}$ del mar (Gómez Otero et al. 2017a). En el sector costero los xilópalos están disponibles en forma de pequeños rodados $(<4 \mathrm{~cm})$ de calidad regular a muy buena. En las mesetas interiores se observó la presencia de tabletas de $<57 \mathrm{~mm}$ de buena a muy buena calidad (Gómez Otero et al. 2017a). Por su parte, Haller consigna el hallazgo de fragmentos de troncos en los afloramientos de la Fm. Puerto Madryn, aunque aún no fueron detectados en nuestros muestreos (Haller 1981). Los bosques más cercanos se localizan a $200 \mathrm{~km}$ al sur en el valle inferior del río Chubut (zona del Dique Ameghino) y a $180 \mathrm{~km}$ al oeste en Telsen.

Para determinar la composición de elementos y explorar potenciales fuentes de procedencia, se realizó un estudio de nueve muestras pequeñas $(<50 \mathrm{~mm})$ de xilópalos: cinco de origen arqueológico y tres paleontológicas (Banegas et al 2016). Las muestras, estudiadas por R. Pujana en el laboratorio del MCNA, fueron primero analizadas bajo

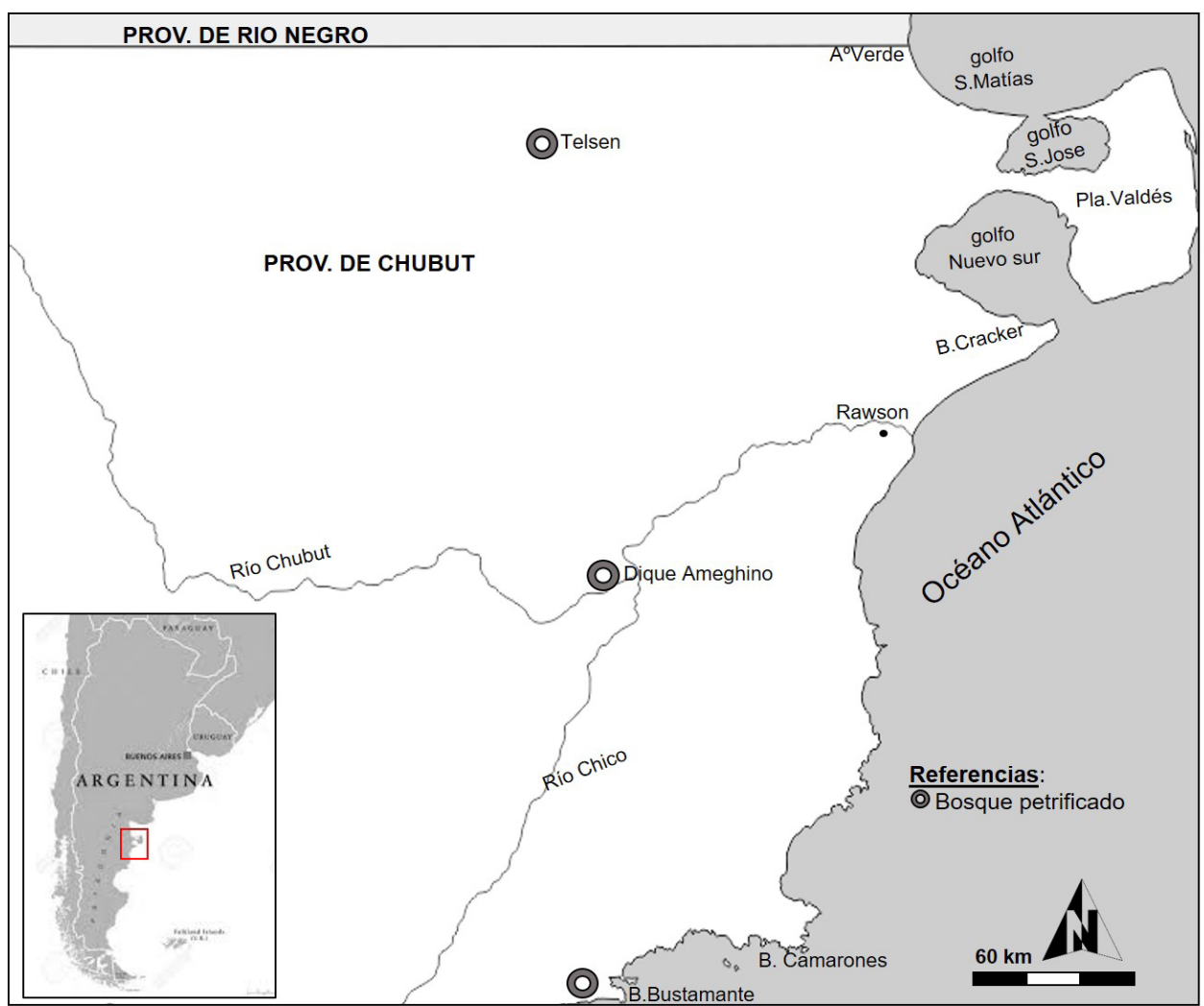

Figura 1. Costa norte y sur de Chubut y localización de los afloramientos de maderas fósiles conocidos.

Figure 1. North and south coast of Chubut and the location of known fossil woods. 
Tabla 1. Sitios arqueológicos y cronología de los contextos analizados.

Table 1. Archaeological sites and chronology of the contexts analyzed.

Tabla 1. Sitios arqueológicos y cronología de los contextos analizados

\begin{tabular}{|c|c|c|c|c|c|c|c|}
\hline \multicolumn{2}{|c|}{ Sitios arqueológicos } & \multirow{2}{*}{$\begin{array}{l}\text { Fechado } \\
7400 \pm 90 \\
\mathrm{C}^{14} \mathrm{AP}\end{array}$} & \multirow{2}{*}{\begin{tabular}{|l|}
$N^{\circ}$ Lab \\
LP-1551 \\
\end{tabular}} & \multirow{2}{*}{$\begin{array}{l}\text { BT } \\
\mathrm{HM}\end{array}$} & \multirow{2}{*}{$\begin{array}{c}\mathrm{N}= \\
\text { líticos } \\
248\end{array}$} & \multirow{2}{*}{$\begin{array}{c}\mathrm{N}= \\
\text { xilópalo }\end{array}$} & \multirow{2}{*}{$\begin{array}{c}\text { Referencias } \\
\text { Gómez Otero } 2006\end{array}$} \\
\hline \multirow{14}{*}{$\begin{array}{l}\text { Costa } \\
\text { Norte }\end{array}$} & $\mathrm{A}^{\circ}$ Verde $1 \mathrm{M} 1$ & & & & & & \\
\hline & Las Lisas $2 \mathrm{CH} 2$ & $\begin{array}{c}2140 \pm 50 \\
C^{14} A P\end{array}$ & LP-875 & $\mathrm{HTI}$ & 263 & 12 & Gómez Otero 2006 \\
\hline & El Progreso $3 \mathrm{M} 3$ & cerámica & -- & HTI & 182 & 20 & Gómez Otero 2006 \\
\hline & La Armonía M2 & $\begin{array}{c}470 \pm 45 \\
C^{14} A P\end{array}$ & LP-969 & HTF & 455 & 28 & Gómez Otero 2006 \\
\hline & Flechero $39 \mathrm{M} 2$ & $\begin{array}{c}2649 \pm 40 \\
\mathrm{C}^{14} \mathrm{AP}\end{array}$ & LP-1022 & $\mathrm{HTI}$ & 193 & 11 & Gómez Otero 2006 \\
\hline & San Román 1 & cerámica & -- & HTF & 172 & 5 & Gómez Otero 2006 \\
\hline & San Pablo 6 C2 M1 & $\begin{array}{c}400 \pm 50 \\
\mathrm{C}^{14} \mathrm{AP}\end{array}$ & LP-2807 & HTF & 86 & 7 & $\begin{array}{c}\text { Gómez Otero et al. } \\
2016\end{array}$ \\
\hline & San Pablo Xil C1 & -- & -- & HTF & 31 & 31 & $\begin{array}{c}\text { Gómez Otero et al. } \\
2016\end{array}$ \\
\hline & Cormoranes $3 \mathrm{~F} 1$ & $\begin{array}{c}4340 \pm 70 \\
\mathrm{C}^{14} \mathrm{AP}\end{array}$ & LP-1085 & $\mathrm{HM}$ & 15 & 1 & Gómez Otero 2006 \\
\hline & Las Ollas 1 & $\begin{array}{l}610 \pm 60 C^{14} \\
A P, 640 \pm 60 \\
\quad C^{14} A P\end{array}$ & $\begin{array}{l}\text { LP-819 } \\
\text { LP-834 }\end{array}$ & HTF & 81 & 2 & Gómez Otero 2006 \\
\hline & Bahía Craker 8 & $\begin{array}{c}5500 \pm 80 \\
\text { AP } 4435 \text { AC- } \\
4081 \text { AC }\end{array}$ & LP-2244 & $\mathrm{HM}$ & 84 & -- & $\begin{array}{l}\text { Gómez Otero et al. } \\
2013\end{array}$ \\
\hline & Bahía Craker 4 & $\begin{array}{l}5390 \pm 130 \\
\text { AP } 4284 \text { AC }\end{array}$ & LP-2324 & $\mathrm{HM}$ & 165 & 1 & $\begin{array}{c}\text { Gómez Otero et al. } \\
2013\end{array}$ \\
\hline & El Pedral $1 \mathrm{M} 1$ & Sin datos & -- & $\mathrm{HTI}$ & 40 & 6 & Banegas y Goye 2015 \\
\hline & Ecoccentro F1 & $\begin{array}{c}840 \pm 50 \\
\mathrm{C}^{14} \mathrm{AP}\end{array}$ & LP-1753 & HTF & 37 & 2 & Gómez Otero 2006 \\
\hline \multirow{4}{*}{$\begin{array}{l}\text { Costa } \\
\text { Sur }\end{array}$} & El Medanal & cerámica & -- & HT & 326 & 96 & $\begin{array}{c}\text { Gómez Otero y Paz } \\
1995\end{array}$ \\
\hline & $\begin{array}{c}\text { La Pingüinera } 1 \mathrm{M} 1 \\
\text { y subm1 }\end{array}$ & $\begin{array}{c}2530 \pm 60 \\
\mathrm{C}^{14} \mathrm{AP}, \mathrm{Cal} \\
2130 \pm 60 \\
\mathrm{C}^{14} \mathrm{AP}\end{array}$ & LP 3559 & HT & 120 & 41 & $\begin{array}{c}\text { Banegas y Aguilera } \\
2019\end{array}$ \\
\hline & La Pingüinera $1 \mathrm{M} 2$ & Sin datos & -- & HT & 119 & 44 & $\begin{array}{c}\text { Banegas y Aguilera } \\
2019\end{array}$ \\
\hline & Restinga Alí & cerámica & -- & HT & 1457 & 583 & Arrigoni y Paleo 1991 \\
\hline
\end{tabular}

microscopio de barrido electrónico (MEB) y luego se utilizó la técnica de detector de energía dispersiva EDX (Energy Dispersive $X$-ray). Los ejemplares arqueológicos habían sido recuperados en muestreos sub-superficiales de los sitios San Pablo taller de xilópalo C1 Nivel 1 (pieza N²6) (Gómez Otero et al. 2017b) y La Azucena 2 Conchero $1 \mathrm{M} 1$ ( $\left.\mathrm{N}^{\circ} 21\right)$, y también en superficie en los sitios Los Abanicos B2 M1 (N45), La Armonía M3 (N9), San Román $1\left(\mathrm{~N}^{\circ} 6\right)$ y Punta Buenos Aires 2 (MPta. Bs-As2) (Gómez Otero 2007). Las tres piezas paleontológicas provienen respectivamente de la Fm. Puerto Madryn (MFmPM), del Bosque Petrificado Ormaechea -zona de los lagos Musters y Colhue Huapi- y de Las Plumas -valle medio del Chubut- (MFmPtCh). Los resultados del estudio bajo MEB determinaron que los ocho ejemplares corresponden a maderas de gimnospermas, aunque la mala preservación de las muestras no permitió la clasificación taxonómica a nivel de género (Banegas et al. 2016). El análisis de composición mediante EDAX, realizado a sólo cinco de los ejemplares - dos paleontológicos (MFmPM y MFmPtCh) y tres arqueológicos ( ${ }^{\circ} 6, N^{\circ} 26$ y MPta. Bs-As2)-, mostró alto grado de permineralización (entre $42 \%$ y 47\%) en todas las muestras. Para el caso de los tres artefactos, los distintos porcentajes de elementos identificados indicarían que se trata de fuentes primarias diferentes, aunque por el momento no se pudo determinar el origen (Banegas et al. 2016).

Costa sur (en adelante CS): Se trata de una costa recortada de más de $180 \mathrm{~km}$ de longitud caracterizada por la presencia de bahías, caletas y numerosas islas e islotes en donde se intercalan ambientes rocosos del Complejo Marifil con cordones litorales y sustratos blandos (Yorio et al. en Crespo 2013). De acuerdo con la información recopilada de las hojas geológicas 4556 II y IV -Camarones- (Camacho 1979, Lema et al. 2001), el área está dominada por afloramientos de la Fm. Marifil (Malvicini y Llambías 1974) de edad Jurásica, conformada 
por riolitas, ignimbritas, tobas y brechas. A ellas se suman depósitos de gravas del Cuaternario que cubren las superficies de pedimentación, y cordones litorales que se ubican en distintas cotas -desde los 15 a 150 msnm(Weiler en Crespo 2013). Al Holoceno más reciente corresponden las acumulaciones coluviales, las vinculadas a la red de drenaje actual y los depósitos asociados al cordón litoral más moderno. Esta costa además se destaca por la oferta de de arroyos que acarrean rodados de tamaño grande (guijón). Con relación a las fuentes de xilópalos, al sur de la localidad de Camarones, se conocen por lo menos dos: a 65 km en bahía Bustamante llamada "La Pirámide" y otra a 130 km en Puerto Visser (Brea y Zucol 2006), que Arrigoni y Paleo (1991) denominaron "Bosque Petrificado de la Costa" (Figura 1). Por su

Tabla 2. Grupos y subgrupos tipológicos de la costa norte y sur de la provincia de Chubut.

Table 2. Typological groups and subgroups of the north and south coast of the province of Chubut.

\begin{tabular}{|c|c|c|}
\hline Grupos y subgrupos & Costa Norte & Costa sur \\
\hline Núcleo & 6 & 51 \\
\hline Flc.núc & 3 & -- \\
\hline Raspador & 7 & 27 \\
\hline Raedera & 2 & -- \\
\hline Pta. Buril & 9 & -- \\
\hline Pta.dest & -- & 27 \\
\hline Pref.bif. & 1 & -- \\
\hline Pta.Proy. & 3 & 6 \\
\hline Muesca & 3 & 21 \\
\hline Dentic. & -- & 21 \\
\hline Gubia & -- & 6 \\
\hline Filo retoc. & 8 & 20 \\
\hline Rst.Comp. & 8 & 81 \\
\hline Lsc.Grde. & 10 & -- \\
\hline Lasc.Med. & 12 & 68 \\
\hline Lasc.Peq. & 16 & 97 \\
\hline Microlasc. & 26 & 171 \\
\hline Hipermic. & 6 & 77 \\
\hline Lám.Grde. & 3 & -- \\
\hline Lám.Med. & 5 & 49 \\
\hline Lám. Peq & 3 & -- \\
\hline Microlám. & -- & 24 \\
\hline
\end{tabular}

parte, Romero (1970) analizó un tronco de madera fósil hallado de manera aislada en bahía Solano y determinó su correspondencia con la especie UIminium atlanticum.

A los fines de iniciar la construcción de la base regional de recursos líticos, se realizaron cuatro muestreos sistemáticos en cordones litorales emplazados entre Camarones y Cabo Dos Bahías (Figura 1). Las litologías que predominan son las sílices (29\%), seguidas de las andesitas (13\%) y las dacitas (11\%), de calidad buena a regular. Respecto de los xilópalos, sólo alcanzaron el 2,5\% y son todos de muy buena calidad (Banegas y Aguilera 2019)

\section{Materiales y métodos}

Para evaluar el tipo y grado de aprovechamiento de los xilópalos, se analizaron conjuntos artefactuales provenientes de sitios publicados que cuentan con datos cronológicos directos (dataciones radiocarbónicas) o indirectos ${ }^{1}$. El total incluye 15 conjuntos líticos de la costa norte $(\mathrm{N}=2054)$ datados entre el Holoceno medio y el tardío final y cuatro de la costa sur $(\mathrm{N}=2074)$ del Holoceno tardío (Tabla 1). Estos conjuntos fueron estudiados siguiendo los lineamientos de Aschero (1975, 1983). Se determinaron las siguientes variables: grupos y subgrupos tipológicos; tamaños, índices de laminaridad y bifacialidad, y calidad de la materia prima según Aragón y Franco (1997).

\section{Resultados}

En el total de conjuntos líticos de $\mathrm{CN}$ se observó la presencia de 131 artefactos de xilópalos $(6,4 \%)$, de muy buena a excelente calidad (Tabla 1). La estructura tipológica está dominada por los desechos de talla (61\%), seguidos por los artefactos formatizados por retoque $(26 \%)$, los de rastros complementarios $(8,4 \%)$ y los núcleos (3,8\%). Entre los instrumentos prevalecen las formas-base laminares (66\%), sobresaliendo los filos retocados laterales simples y dobles (25\% y 15\%) seguidos por los raspadores (18\%). La bifacialidad alcanza el $5 \%$ y está representada en puntas de proyectil y una preforma bifacial (Tabla 2). En cuanto a los tamaños, los núcleos provienen de rodados mediano-pequeños (la mayoría agotados), y también de fragmentos tabulares grandes. Los desechos se distribuyen entre tamaños mediano-grandes (20\%), mediano-pequeños (25\%) y muy pequeños (22\%) (Tabla 2).

En el total de los conjuntos líticos de la CS, se identificaron $752(36,3 \%)$ artefactos de xilópalo de muy buena a excelente calidad (Tabla 1). La estructura está compuesta por desechos de talla (65\%), seguidos por los artefactos retocados por talla (18\%), los de rastros

${ }_{1}^{1}$ Para el caso de los muestreos en estratigrafía se tuvo en cuenta el fechado radiocarbónico, mientras que para los contextos de superficie se tomaron en cuenta indicadores cronológicos indirectos como, por ejemplo, la presencia de cerámica. 
complementarios (11\%) y los núcleos (7\%). Los subgrupos más representados son las puntas destacadas y los raspadores (20\% cada uno), y en segundo orden de importancia, los filos laterales retocados simples y dobles, los denticulados y las muescas (15\% cada uno) (Tabla 2). Entre los instrumentos, los soportes laminares alcanzan un $18 \%$ y la bifacialidad sólo el 1,8\%, que está representada solo en puntas de proyectil. Los núcleos son medianos y medianos-pequeños, y el tipo que prevalece es el de láminas. Entre los desechos predominan las lascas muy pequeñas (entre 35\% y 15\%) (Tabla 2).

Si se compara la estructura tipológica de ambas costas se observa que los núcleos y los rastros complementarios están más representados en la CS (7\% y $11 \%)$ que en la CN (3,8\% y 8,4\%). También se registraron diferencias entre las frecuencias y subgrupos tipológicos: en la CN prevalecen los filos retocados laterales simples y dobles (25\% y $15 \%$ ) seguidos por los raspadores (18\%), mientras que en la CS son mayoría las puntas destacadas y los raspadores (20\% cada uno); seguidos por los filos retocados laterales simples y dobles; los denticulados y las muescas (15\% cada uno). En cuanto a las tendencias temporales, para la costa norte se determinaron cambios en la frecuencia de uso de los xilópalos: nula a muy baja en los contextos del Holoceno medio (0 a 6,6\%), un incremento leve en el Holoceno tardío inicial (3 a 15\%) y una leve merma en el Holoceno tardío final (2,5 a 8\%) (Banegas et al. 2016). Esta tendencia no pudo evaluarse para la costa sur porque todos los contextos fueron datados o asignados al Holoceno tardío (Tabla 1).

\section{Discusión y conclusiones preliminares}

En ambos sectores la calidad de los nódulos de los xilópalos utilizados es muy buena a excelente; en cuanto al tamaño, son pequeños a mediano-grandes en la CN y mediano-grandes a grandes en la CS. Se calculó el promedio total de ocurrencia de artefactos de xilópalo para cada área: en CN, 131 (8,7 promedio artefactos/ sitio); en CS, 752 (188 promedio/sitio), lo que señala un aprovechamiento muy bajo en el sector norte y alto en el sur. Estas diferencias se vincularían con la oferta local (forma, tamaño, abundancia) y la calidad para la talla en cada área. Como se señaló arriba, en la costa norte y mesetas adyacentes los xilópalos son de muy buena calidad, pero su disponibilidad es muy baja $(1,5 \%)$ y se presentan en forma de guijarros o tabletas pequeñas. Sin embargo, la proporción es mayor en los conjuntos artefactuales (entre 5\% y 32\%) y el tamaño de los instrumentos supera al de los nódulos locales. Esto indica dos alternativas: el uso de fuentes primarias alejadas (posiblemente la del Dique Ameghino y/o la de Telsen), o también la explotación de fragmentos más grandes hallados fortuitamente en los afloramientos del Terciario. Por su parte, en la costa sur la presencia de rodados de xilópalos es también muy baja (2,5\%), pero están los dos bosques de xilópalos de excelente calidad (el de bahía
Bustamante y el de Puerto Visser) separados por 70 km; además hay disponibilidad de troncos transportados por acción hídrica desde las mesetas altas. Esto también explicaría la alta representación de núcleos y el tamaño mayor de los artefactos en CS respecto de CN. Un aspecto llamativo es la notoria mayor abundancia de los soportes laminares en la CN (66\%) que en la CS (18\%), lo que sugiere la aplicación de una estrategia para optimizar el aprovechamiento y transporte de esta roca no local a partir de soportes con mayor porcentaje de filos útiles. Por último, la bifacialidad fue poco registrada en ambos sectores lo que, como se propuso antes, podría vincularse con la estructura interna de los xilópalos (presencia de nudos) que limitaría la talla de artefactos mediante esta técnica (Banegas et al. 2016).

A manera de conclusión, para ambos sectores costeros este estudio indica una vinculación estrecha entre la intensidad de uso de los xilópalos y la oferta local: a menor distancia respecto de los afloramientos primarios de excelente calidad, mayor aprovechamiento, y viceversa. En consecuencia, se infieren dos situaciones diferentes: a) los grupos de la costa sur habrían explotado intensamente y de manera directa los bosques petrificados y también los troncos aislados acarreados por los arroyos locales; b) los de la costa norte podrían haber aprovechado los hallazgos ocasionales de troncos en los afloramientos del Terciario y también haber obtenido nódulos por intercambio o por adquisición directa en los bosques más cercanos. La agenda futura plantea integrar y discutir estos resultados con los de estudios de procedencia de otras rocas como obsidianas o calcedonias de filón. Esto permitirá, comparar y enriquecer el conocimiento sobre los circuitos y vectores de circulación de estas materias primas de excelente calidad y atributos visuales destacados como color, textura y brillo.

Puerto Madryn, Chubut.

\section{Agradecimientos:}

A los editores Fabiana Skarbun, Manuel Cueto y Ariel Frank por brindarnos la posibilidad de participar en este volumen especial. A los dos evaluadores anónimos por sus comentarios y sugerencias. Este trabajo se realizó con el financiamiento de CONICET, y con los fondos de los proyectos PIP 11220130100308 y PID 1254, otorgados a Julieta Gómez Otero.

\section{Referencias Citadas}

Ambrústolo, P. 2010 Estudio de las estrategias de aprovisionamiento y utilización de los recursos líticos por grupos cazadores-recolectores en la costa norte de Santa Cruz (Patagonia Argentina). Tesis Doctoral inédita, Facultad de Ciencias Naturales y Museo, Universidad Nacional de La Plata.

Arrigoni, G. y C. Paleo. 1991. Investigaciones arqueológicas en 
la región central del golfo San Jorge (desde Punta Peligro, Pcia. del Chubut hasta el límite con la Pcia. de Santa Cruz). Shincal 3, T.3: 206-210. Catamarca.

Aschero, C. 1975-1983 Ensayo para una clasificación morfológica de artefactos líticos aplicada a estudios tipológicos comparativos. Informe al CONICET. Revision 1983. MS.

Banegas, A. y S. Goye. 2014. Spatial and temporal variability in the use of lithic raw materials for flaked stone technology in northeast Chubut Province (North Patagonia) during the Late Holocene. En Archaeology of coastal hunter-gatherer occupations in the Southrn Cone, editado por P. Ambrústolo y M.A. Zubimendi, Quaternary International Vol. 373:55-62.

Banegas, A y. Aguilera. 2019. Base regional de recursos líticos y selección de materias primas en Bahía Camarones. En Arqueología de la Patagonia: el pasado entre las arenas. Gómez Otero, Svoboda y Banegas (eds).

Banegas, A., S. Goye y J. Gómez Otero. 2015. Caracterización regional de recursos líticos en el nordeste de la provincia del Chubut (Argentina). En Materias primas líticas en Patagonia. Localización, circulación y métodos de estudio de las fuentes de rocas de la Patagonia argentino-chilena, eds. J. Alberti and V. Fernández, Intersecciones en Antropología, Dossier 2: 39-50.

Banegas, A., R. Pujana y J. Gómez Otero. 2016. Caracterización tecnológica de xilópalos de la costa centro-septentrional de Patagonia: tendencias temporales y potenciales fuentes de aprovisionamiento. Arqueología de Patagonia: De mar a mar, Actas de las IX Jornadas de Arqueología de la Patagonia. Pp. 415-422. Coyaique, Chile.

Brea, M. y A. Zucol. 2006. Leños fósiles de Boraginaceae de la Formación Peñas Coloradas (Paleoceno superior), Puerto Visser, Chubut, Argentina. En AMEGHINIANA (Rev. Asoc. Paleontol. Argent.) - 43 (1): 139-146. Buenos Aires, 30-03-2006

Camacho, H. 1979. Descripción Geológica de la Hoja 47h-48g, Bahía Camarones. Servicio geológico Nacional.

Crespo, E. (Compilador). 2013. Documento Borrador de Caracterización Inicial del Parque Interjursdiccional Marino Costero Patagonia Austral. Chubut, Equipo Técnico de Planificación Interjurisdiccional APN/ANP del CHUBUT. Ms.

Gómez Otero, J. 2006. Dieta, uso el espacio y evolución en poblaciones cazadoras-recolectoras de la costa centroseptentrional de Patagonia durante el Holoceno medio y tardío. Tesis doctoral inédita, Universidad Nacional de Buenos Aires.

Gómez Otero, J. y M. J. Paz. 1994. Análisis tipológico y tecnomorfológico de materiales líticos del sitio costero "El Medanal" (Prov. del Chubut). Revista del Museo de Historia Natural de San Rafael XIV (1-4): 298-300.
Gómez Otero, J., J. Belardi, A. Súnico y R. Taylor. 1999 Arqueología de cazadores-recolectores en península Valdés (costa central de Patagonia): primeros resultados. En Soplando en el Viento, editado por J. B. Belardi, P. Fernández, R. Goñi, G. Guráieb y M. De Nigris, pp. 393-417. Instituto Nacional de Antropología y Pensamiento Latinoamericano, Universidad Nacional del Comahue, Neuquén.

Gómez Otero, J. V. Schuster y A. Banegas. 2017a. Archaeology of the Península Valdés: spatial and temporal variability in the human use of the landscape and geological resources. En Late Cenozoic of Península Valdés: an interdisciplinary approach. Editores Pablo Bouza y Bilmes Andrés. Springer Nature. (pp. 1-54). Reino Unido, Londres.

Gómez Otero, J., A. Banegas, L. Caruso Fermé; M.S. Goye, G. Millán; V. Schuster; A. Svoboda y N. Weiler. Los antiguos pobladores humanos: arqueología de la Bajada Colombo. En: Reserva de Vida Silvestre San Pablo de Valdés: 10 años conservando el patrimonio natural y cultural de la Península Valdés, Patagonia Argentina., editado por D. Udrizar Sauthier, G. Pazos y A. Arias, pp.229-247. Fundación Vida Silvestre, Buenos Aires.

Haller, M. 1981 Descripción Geológica de la Hoja 43h- Puerto Madryn, Boletín Servicio Geológico Nacional Nº. 184:41.

Haller, M. 2017. Geology of Península Valdés. En Late Cenozoic of Península Valdés: an interdisciplinary approach. Editores Pablo Bouza y Bilmes Andrés. Springer Nature. (pp. 23-46). Reino Unido, Londres.

Lema, H., Busteros, A. y Franchi M. 2001. Hoja Geológica 4566 II y IV. Camarones, Provincia del Chubut. Boletín 261. Servicio Geológico y Minero Argentino.

Malvicini, L. y E. Llambías. 1974 Geología y génesis del depósito de manganeso Arroyo Verde, provincia del Chubut, República Argentina. Actas $\vee$ Congreso Geológico Argentino, tomo II: 185-202, Buenos Aires.

Mansur, E. 2008. Arqueología de la zona de Punta Bustamante (Prov. De Santa Cruz, Argentina). En Arqueología de la costa patagónica. Perspectivas para la conservación, editado por Isabel Cruz y María Soledad Caracotche, pp. 173-193, Universidad Nacional de la Patagonia Austral-Subsecretaría de Cultura de la Provincia de Santa Cruz, Río Gallegos.

Massaferro, G. y Haller, M. 2000. Texturas de las vetas epitermales del Macizo Norpatagónico. $5^{\circ}$ Congreso de Mineralogía y Metalogenia, Actas: 312-319, La Plata.

Romero, E. J. 1970. Ulminium atlanticum n.sp. tronco petrificado de Laurácea del Eoceno de Bahía Solana, Chubut, Argentina. Ameghiniana 7 (3): 205-224. Buenos Aires. 